Vera-Estrada, F.; Sánchez-Rivas, E. y Sánchez-Rodríguez, J. (2018) Promoción de la actividad física saludable en el recreo escolar / Promotion of Healthy Physical Activity in School Recreation. Revista Internacional de Medicina y Ciencias de la Actividad Física y el Deporte vol. 18 (72) pp. 655-668 Http://cdeporte.rediris.es/revista/revista72/artpromocion980.htm

DOI: http://doi.org/10.15366/rimcafd2018.72.004

\title{
ORIGINAL
}

\section{PROMOCIÓN DE LA ACTIVIDAD FÍSICA SALUDABLE EN EL RECREO ESCOLAR}

\section{PROMOTION OF HEALTHY PHYSICAL ACTIVITY IN SCHOOL RECREATION}

\author{
Vera-Estrada, F. ${ }^{1}$; Sánchez-Rivas, E. ${ }^{2}$ y Sánchez-Rodríguez, J. ${ }^{3}$ \\ 1 Profesor Primaria. CEIP José María Hinojosa de Málaga (España) fveraestrada@gmail.com \\ ${ }^{2}$ Asesor de formación. Centro del Profesorado de Málaga (España) enriquesr@cepmalaga.com \\ 3 Profesor Titular Universidad. Facultad de Ciencias de la Educación. Universidad de Málaga \\ (España) josesanchez@uma.es
}

Agradecimiento: los autores desean mostrar su agradecimiento al profesorado de Educación Física de los centros participantes por su colaboración, a los equipos directivos de dichos centros por su apoyo y a las familias de los alumnos por autorizar la realización de la investigación.

Código Unesco: 5599 Otras especialidades pedagógicas (Educación Física y Deportes) / Other pedagogy specialities (Physical Education and Sports)

Clasificación del Consejo de Europa: 4 Educación Física y Deporte comparado / Physical Education and comparative

Sports.

Recibido 8 de febrero de 2017 Received February 8, 2017

Aceptado 17 de mayo de 2017 Accepted May 17, 2017

\section{RESUMEN}

El objetivo de este trabajo fue investigar qué medidas se podrían tomar para aumentar los niveles generales de actividad física del alumnado de sexto de Educación Primaria durante los periodos de recreo escolar. Se implementó un programa específico desde el currículum de la asignatura de Educación Física y, después de haber finalizado la aplicación del citado programa, se comprobó los niveles de actividad física. También se realizó una observación pasados treinta días de su finalización, con el fin de comprobar en qué medida se modifican los hábitos de actividad física. El instrumento de recogida de datos fue el podómetro, que los participantes utilizaron durante los periodos de recreo escolar. El análisis de los resultados permite afirmar que la aplicación del programa específico objeto de estudio incide positivamente sobre el incremento del volumen de actividad física que realiza el alumnado en su tiempo libre y que, 
pasados treinta días desde su aplicación, el alumnado mantiene parte de incremento adquirido, modificando su hábito de actividad durante el recreo escolar.

PALABRAS CLAVE: salud pública; actividad física; niños; escuelas; recreo.

\begin{abstract}
The objective of this work was to investigate what measures could be taken to increase the general levels of physical activity of students in sixth grade of Primary Education during the school breaks. A specific program was implemented from the curriculum of the subject of Physical Education and, after completing its application, physical activity levels were analyzed. In addition, an observation was conducted thirty days after its completion, in order to verify to what extent the habits of physical activity are modified. The instrument of data collection was the pedometer, which the participants used during the periods of school break. The analysis of the results allows affirming that the application of the specific program object of study affects positively on the increase of the volume of physical activity that the students do in their free time and that, after thirty days from its application, the students keep part of this increase acquired, which means a change in their activity habit during school breaks.
\end{abstract}

KEY WORDS: public health, physical activity, children, schools, recess.

\title{
INTRODUCCIÓN
}

La educación para la salud y la actividad física ha ido cobrando protagonismo en las políticas educativas, que promueven el desarrollo de hábitos y estilos de vida más saludables en el alumnado a través del currículum escolar. Con ello se busca reducir los crecientes índices de sendentarismo y sobrepeso infantil (OMS, 2017; World Health Organization, 2010).

Estas nuevas demandas educativas recomiendan conocer mejor la incidencia de la actividad física sobre la población en edad escolar. En esta línea Tudor-Locke et al. (2011) demuestran que el volumen saludable para sujetos de entre ocho y diez años de edad se sitúa entre los 12.000 y 16.000 pasos al día. En relación con el tiempo escolar, se aprecia un progresivo descenso desde el intervalo saludable, que se alcanza en la edad infantil, hasta valores medios de entre 8.000 y 9.000 pasos/día en adolescentes (Tudor-Locke, Johnson y Katzmarzyk, 2010). 
Tabla 1: cuantificación de pasos diarios necesarios para la mejora de la salud en niños y niñas entre seis y doce años

\begin{tabular}{|l|l|}
\hline Los valores para los niños son: & Los valores para las niñas son: \\
\hline$<10000 ;$ "sedentarios" & $<7,000$; "sedentarias" \\
\hline $10,000-12,499 ;$ "bajo en activo" & $7,000-9,499 ;$ "bajo en activo" \\
\hline $12,500-14,999 ;$ "poco activo" & $9,500-11,999 ;$ "poco activa" \\
\hline $15.000-17.499$, "activo" & $12.000-14.499 ;$ "activa" \\
\hline$\geq 17.500$ pasos / día; "muy activo" & $\geq 14.500$ pasos / día; "muy activa" \\
\hline \multicolumn{2}{|c|}{ Fuente: Tudor-Locke, Hatano, Pangrazi, \& Kang (2008) } \\
\hline
\end{tabular}

Diversas investigaciones han demostrado la importancia de los periodos de recreo escolar para alcanzar un volumen de actividad física saludable. Guinhouya et al. (2006) establecieron que el recreo aporta hasta el $26 \%$ de la actividad física diaria, moderada y vigorosa de los jóvenes en edad escolar. Ridgers, Stratton y Fairclough (2006) sugirieron que el recreo escolar puede contribuir entre un $5 \%$ y $40 \%$ de la actividad física diaria recomendada; y que el recreo colabora entre un $6 \%$ y un $13 \%$ de la actividad física moderada-intensa que debe acumular diariamente los niños de ocho a once años (Ridgers, Tóth y Uvacsek, 2009).

El recreo, junto con las sesiones de educación física, resulta clave para la promoción de la actividad física hasta niveles saludables (Fairclough, Beighle, Erwin, \& Ridgers, 2012; Martínez Martínez, Contreras Jordán, Lera Navarro, \& Aznar Laín, 2012; Pérez López \& Delgado Fernández, 2013; Ridgers, Stratton, \& Fairclough, 2005; Ridgers et al., 2006; Stratton, 1999; World Health Organization, 2010).

Teniendo como referencia la literatura específica sobre el desarrollo de la actividad física en el recreo escolar, la mayor parte de estudios se circunscriben a la variable personal de género. Ridgers, Saint-Maurice, Welk, Siahpush y Huberty (2011); Beighle, Morgan, Le Masurier y Pangrazi (2006) y Ridgers et al. $(2011,2009)$ constataron que, con una actividad libremente elegida, los niños acumulan más actividad física que las niñas durante el periodo de recreo escolar. En esta línea también se encuadran distintos trabajos (Aznar et al., 2011; Haug, Torsheim, \& Samdal, 2010; Martínez, Aznar, \& Contreras, 2015; Sarkin, McKenzie, \& Sallis, 1997; Tudor-Locke et al., 2011).

Otras líneas de investigación han profundizado sobre la relación de la actividad física en periodos de recreo escolar con el nivel educativo (FaisonHodge y Porretta, 2004; Haug et al., 2010), la edad (Ridgers et al., 2005; Ridgers, Stratton y McKenzie, 2010) o el índice de masa corporal (McKenzie et al., 2010; Gareth et al., 2007).

Sin embargo, no se han encontrado estudios que analicen si el volumen de actividad física del alumnado en el recreo se incrementa después de la aplicación de programas específicos vinculados a la asignatura de educación física. Los estudios más próximos comparan el volumen de actividad entre la 
clase de educación física y el recreo escolar (Escalante, Backx, Saavedra, García-Hermoso, \& Domínguez, 2011; Jennings-Aburto et al., 2009; Sarkin et al., 1997).

Esto recomienda orientar el trabajo hacia la determinación del impacto que tiene un programa específico diseñado en el marco del presente estudio (Sánchez-Rivas y Vera-Estrada, 2013) sobre la actividad que el alumnado realiza de forma libre en un periodo de ocio, como es el recreo escolar. Con ello, el objetivo propuesto se concreta en conocer si, tras la aplicación de un programa específico para el fomento de la actividad física recreativa a través de las clases de Educación Física, aumenta el volumen de actividad física del alumnado participante durante los periodos de recreo escolar.

En el caso de que se produzca un aumento del volumen de actividad tras aplicar el programa, se profundizará en el conocimiento sobre el nivel de consolidación del incremento de actividad. Para ello, resulta imprescindible conocer en qué medida se mantienen los incrementos obtenidos después de pasados treinta días de la finalización del periodo de implementación de un programa específico para el fomento de la actividad física recreativa.

\section{MATERIAL Y MÉTODOS}

\section{Población y muestra}

La población está compuesta por alumnos de centros educativos públicos y concertados que imparten Educación Primaria en el ámbito territorial de un distrito de la ciudad de Málaga. Se realiza, por tanto, un muestreo no probabilístico (accidental) determinado por la facilidad de acceso a los datos de contacto, a través de los listados que el Ayuntamiento de Málaga publica en su portal institucional. Con ello, el filtrado de los centros educativos participantes se articula según un criterio de accesibilidad según su proximidad. Así, se obtuvo una muestra de 10 centros (con 492 sujetos).

Dado que el programa diseñado se dirige al alumnado de sexto de Educación Primaria, a efectos de población y muestra se considera al alumnado que cursa este nivel durante el periodo de investigación (tabla 2).

Tabla 2: participantes

\begin{tabular}{|c|c|c|c|c|c|}
\hline & $\begin{array}{c}\text { Población } \\
\text { (distrito de } \\
\text { Málaga) }\end{array}$ & $\begin{array}{c}\text { Colegios } \\
\text { conectados }\end{array}$ & Muestra & $\begin{array}{c}\text { Grupo } \\
\text { experimental } \\
\left(6^{\circ} \mathrm{A}\right)\end{array}$ & $\begin{array}{c}\text { Grupo } \\
\text { Control }\left(6^{\circ}\right. \\
\mathrm{B})\end{array}$ \\
\hline Colegios & 18 & 18 & 10 & 10 & 10 \\
\hline Alumnado & 863 & - & 492 & 243 & 249 \\
\hline
\end{tabular}

Fuente: elaboración propia

La muestra se estructuró en dos grupos. El primero de ellos, al que llamaremos "experimental" está compuesto por todos los sujetos matriculados en la letra $A$, es decir el alumnado de 6ㅇ $A$ de los diez centros conforma el grupo al que se le aplica el programa. El segundo grupo está compuesto por los 
inscritos en la letra $B$, y no recibirán la acción de la medida, actuando como "grupo control". Ninguno de los centros de la muestra tiene más de dos líneas educativas en sexto. En este sentido, cabe reseñar que los diez centros que aceptaron formar parte del estudio tenían una organización dos líneas, como suele ser habitual en la zona escolar del distrito de Málaga seleccionado.

\section{Principios éticos y recogida de datos}

Tomando con referencia la Declaración de Helsinki promulgada por la Asociación Médica Mundial (AMM), se diseñó una serie de pautas para la dirigir la recogida de datos.

Antes de iniciar el trabajo de campo, se procedió a informar a las familias y al alumnado implicado, al que se le entregó una autorización de consentimiento para poder participar en la investigación, la cual debía ser devuelta y firmada por los padres/madres o tutores legales. Con la autorización de consentimiento se incluyó una hoja informativa a los padres, madres y/o tutores legales donde se les indicaba los objetivos de la investigación y los procedimientos a seguir en el estudio. En ella, también se les recordó que la participación es voluntaria y anónima. Para poder aclarar las dudas que pudieran surgir y alentar a la participación se citó a los padres/madres y tutores legales del alumnado de sexto a una reunión informativa.

Una vez obtenido el consentimiento firmado, el profesorado participante en el estudio se reunió para unificar criterios y reducir la presencia de variables significativas en el desarrollo del trabajo de campo (información inicial, tareas del programa, pautas para los recreos, etc.) y la recogida de información. Ya en clase de Educación Física, cada uno de los docentes explicó a su alumnado el protocolo para la toma de datos en la hoja de registro de pasos diarios.

A efectos de mejorar la claridad expositiva, hemos establecidos tres fases en el desarrollo de la secuencia de recogida de datos:

(1) Fase de aplicación del pre-test. Para conseguir una familiarización con el instrumento de recogida de datos, y antes de la aplicación del pre-test, los sujetos de la muestra utilizaron un podómetro durante una semana lectiva (de lunes a viernes) para evitar el efecto novedad (reactividad). Tras el periodo de familiarización, se procedió a la recogida de datos (pretest) durante treinta días lectivos, en los periodos de recreo escolar.

Paralelamente, se estableció un protocolo de uso del podómetro, donde se indicó cómo ponerse y quitarse el podómetro, así cómo y cuándo se anotaban los datos obtenidos. 
Tabla 3: Protocolo de uso del podómetro

1. Los estudiantes se colocarán los podómetros en la cintura durante el recreo, que dura 30 minutos. Este proceso se repetirá de lunes a viernes.

2. Aproximadamente 5 minutos antes del recreo, los investigadores entrarán en las clases (dicha intervención está consensuada con equipo directivo del centro y profesorado que imparte clases en los cursos de sexto en el horario anterior al inicio del recreo) y distribuirán los podómetros a cada participante.

3. Al sonido de la sirena ambas clases se lo colocarán en el lugar indicado y bajarán al recreo, iniciándose en ese momento el registro digitalizado de la cantidad de pasos acumulados durante el dicho período.

4. Al volver a sonar el timbre que indica el final del tiempo de recreo el alumnado se colocará en sus respectivas filas donde los investigadores registrarán los datos pertinentes.

5. Una vez concluido la toma de datos, los podómetros se recogerán y se pondrán a cero para el siguiente registro.

\section{Fuente: elaboración propia}

Una vez habituados al uso del podómetro y establecido el protocolo de uso del mismo se inició la toma de datos (pasos realizados) durante un mes en el período de recreo escolar. No se alteró el transcurso normal del recreo, simplemente se limitó a la recogida de datos tanto al grupo control como al grupo experimental.

(2) Fase de aplicación del programa y del test. El programa de intervención diseñado (Sánchez-Rivas y Vera-Estrada, 2013) se integró en el currículum de la asignatura de Educación Física. Su desarrollo tuvo lugar entre el primer y segundo trimestre del calendario escolar. Desde las sesiones de Educación Física, se les aplicó a los sujetos adscritos al grupo experimental durante un periodo de treinta días lectivos, distribuidos en tres Unidades didácticas (tabla $4)$.

Tabla 4: Contenidos del programa

\begin{tabular}{|c|c|c|}
\hline Unidad didáctica & Sesiones & Contenidos básicos \\
\hline Hanae construye sus materiales & 10 & $\begin{array}{c}\text { Juegos alternativos con material } \\
\text { autoconstruido }\end{array}$ \\
\hline $\mathrm{Ni} \mathrm{Ye,} \mathrm{el} \mathrm{señor} \mathrm{de} \mathrm{los} \mathrm{juegos}$ & 10 & Juegos populares y tradicionales \\
\hline Paula es deportista & 10 & Juegos pre-deportivos \\
\hline
\end{tabular}

Fuente: elaboración propia

(3) Fase de aplicación del post-test. Esta fase implicó una nueva recogida de datos, que tuvo lugar durante treinta días lectivos, y que se realizó un mes después de la finalización del programa de intervención, a lo largo del tercer trimestre del calendario escolar.

\section{Instrumento}

El instrumento para la recogida de datos fue el podómetro Dista Newfeel 100 (Oxylane, Villeneuve d'Ascq cedex, Francia). López Fernández, PascualMartos y Álvarez Carnero (2013) lo consideran una herramienta objetiva y fiable 
para medir la cantidad de actividad física realizada en las actividades cotidianas y concluyen que es un instrumento válido, considerado por la comunidad científica internacional como el instrumento de referencia para el conteo de pasos. Otros trabajos (Schneider, Crouter, y Bassett, 2004; Tudor-Locke et al., 2009) se posicionan en el mismo sentido.

\section{Análisis estadístico}

Una vez finalizado el trabajo de campo se realizó el tratamiento estadístico de los datos con el programa SPSS (programa registrado. Versión 20).

Considerando que nuestra investigación se basa en el análisis de las relaciones entre variables (tabla 5), aplicaremos la prueba "t de Student" para muestras independientes. Esta prueba se complementará con el análisis de los estadísticos descriptivos básicos.

Tabla 5: Principales variables implicadas en el estudio

\begin{tabular}{|l|l|}
\hline Variable independiente & $\begin{array}{l}\text { Programa específico para el fomento de la actividad física } \\
\text { recreativa (Sánchez-Rivas y Vera-Estrada, 2013) }\end{array}$ \\
\hline $\begin{array}{l}\text { Primera variable dependiente } \\
\text { (VD1) }\end{array}$ & $\begin{array}{l}\text { Los parámetros de actividad física acumulada inmediatos } \\
\text { (pasos realizados) del alumnado participante }\end{array}$ \\
\hline $\begin{array}{l}\text { Segunda variable dependiente } \\
\text { (VD2) }\end{array}$ & $\begin{array}{l}\text { Los parámetros de actividad física acumulada mantenidos } \\
\text { (pasos realizados) del alumnado participante }\end{array}$ \\
\hline
\end{tabular}

Fuente: elaboración propia

Previamente, y dado que se trata de una prueba paramétrica, se comprobó el cumplimiento de los requisitos de Normalidad y Homocedasticidad mediante los contrastes de Shapiro-Wilk y Levene respectivamente.

\section{RESULTADOS}

Para determinar el valor adquirido por las variables dependientes se halló la media de pasos tanto para el grupo experimental como para el grupo control en las diversas tomas (tabla 6).

Tabla 6: Valor de las variables dependientes (VD)

\begin{tabular}{|l|l|l|l|}
\hline & Pre-test & Test (VD 1) & Post-test (VD 2) \\
\hline $\begin{array}{l}\text { Grupo experimental (media } \\
\text { de pasos) }\end{array}$ & $2.057,44$ & $2.846,56$ & $2.357,88$ \\
\hline $\begin{array}{l}\text { Grupo control (media de } \\
\text { pasos) }\end{array}$ & $1.701,19$ & $1.995,87$ & $1.818,52$ \\
\hline
\end{tabular}

Fuente: elaboración propia

Como muestran los datos de la tabla 6, el aumento de los niveles generales de actividad física en el recreo escolar que ha obtenido el grupo experimental ha pasado de 2.057,44 pasos de media a los 2.846,56 pasos de media durante la aplicación del programa establecido, por lo que la diferencia entre el pre-test y el test es de 789,12 pasos de media al día, lo que supone un incremento medio del $38,35 \%$. Este elevado porcentaje apunta a la existencia de diferencias significativas asociadas al programa. 
Continuando en un plano meramente descriptivo, cabe reseñar la existencia de diferencias muy importantes entre ambos grupos. Mientras en el pre-test la diferencia de pasos es de 356,25 pasos/día, en el test esa diferencia entre grupo control y grupo experimental aumenta hasta los 850,69 pasos/día, lo que representa un aumento del $138 \%$ con respecto al valor inicial.

Respecto al hábito de actividad física consolidado (en número de pasos) del alumnado, transcurridos treinta días del fin del programa de intervención, se obtienen unos valores que suponen un aumento de 300,44 pasos/día, pasando el volumen de actividad física de $2.057,44$ pasos/día a $2.357,88$ pasos/día.

Porcentualmente, los incrementos obtenidos suponen un nivel de consolidación del hábito de actividad física del $38,07 \%$, que se valora de forma significativa. Si lo analizamos en sentido inverso, se produce un descenso de 488,56 pasos/día, pasando de $2.846,44$ a $2.357,88$, lo que supone una reducción porcentual del $61,93 \%$ a los 30 días del fin del programa

Las diferencias se hacen patentes al revisar los datos en un diagrama de cajas (gráfico 1). Al comparar los post-test de ambos grupos se aprecia que los índices de tendencia central del grupo experimental (2) son superiores a los del grupo control (1). En segundo lugar, al comparar el test y el post-test en cada grupo, podemos contrastar que las cajas del post-test están por debajo de las del test. En tercer lugar, si comparamos las cajas de los post-test con la de los pre-test, en el caso del grupo control está prácticamente igual, mientras que en el grupo experimental sí que existe cierta retención, es decir se aprecia que el índice de tendencia central está claramente por encima del pre-test. En último lugar, si comparamos las posiciones de las cajas en cada grupo, estas nos señalan que en el grupo control son similares en pre-test, test y post-test, mientras que en el grupo experimental sí se aprecian mayores diferencias, siendo mayor entre el pre-test y el test que entre el test y el post-test. 


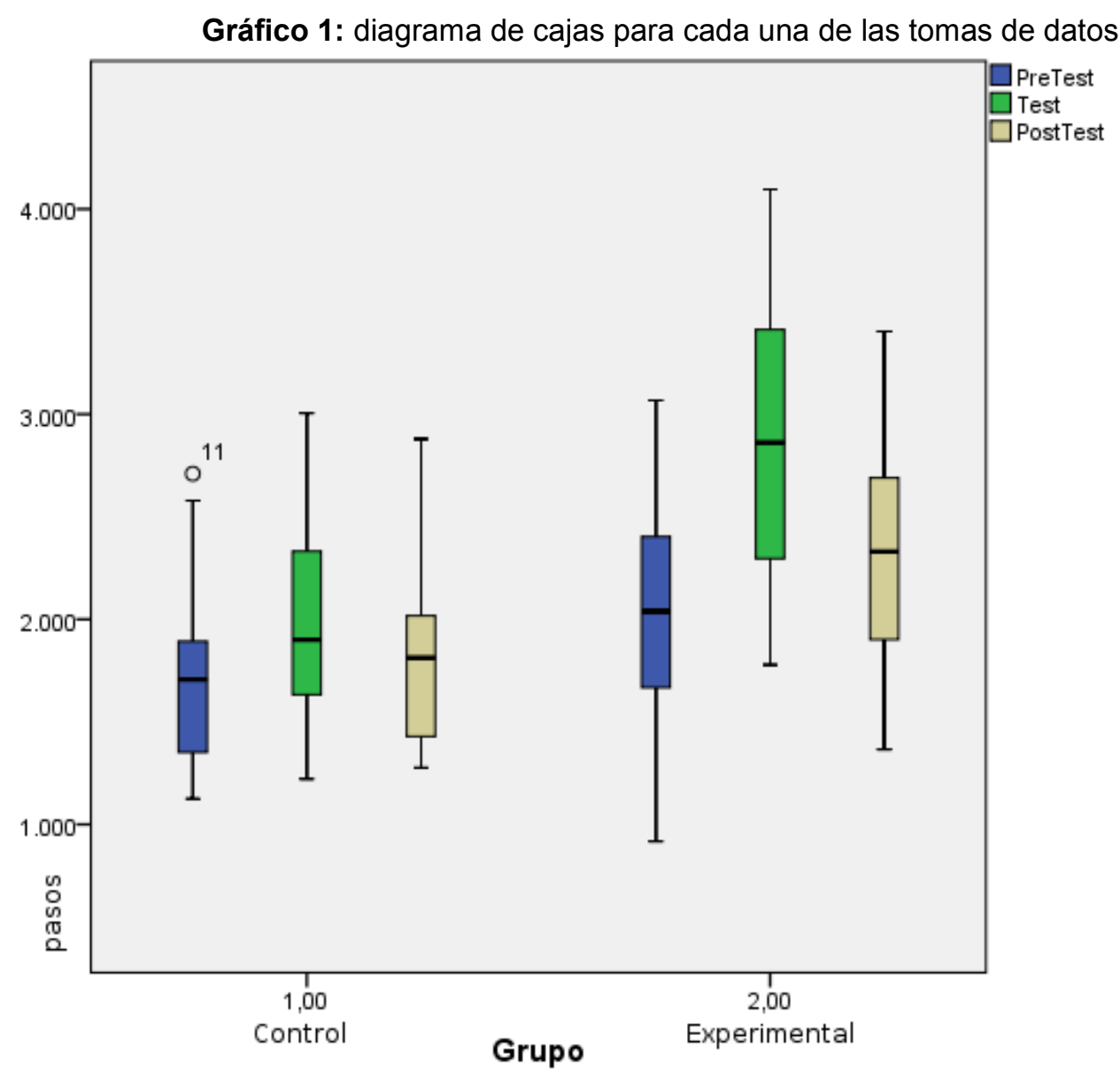

Fuente: elaboración propia

Las diferencias que se aprecian en el análisis descriptivo recomiendan profundizar en la estadística inferencial. Antes de aplicar la prueba la prueba paramétrica t-student para muestras independientes, es pertinente realizar contrastes de Normalidad. Dado el tamaño de la muestra, se aplica la prueba de Shapiro-Wilk. El resultado obtenido en la prueba de Shapiro-Wilk es superior a 0,05 (tabla 7), lo que recomienda la aplicación de pruebas paramétricas. 
Tabla 7: Resultados de la prueba de Shapiro-Wilk

\begin{tabular}{|ll|r|r|r|}
\hline \multirow{2}{*}{\multicolumn{2}{|l|}{ Grupo }} & \multicolumn{3}{|c|}{ Shapiro-Wilk } \\
\cline { 2 - 5 } & Estadístico & \multicolumn{1}{|c|}{ gl } & \multicolumn{1}{c|}{ Sig. } \\
\hline Test & 1,00 &, 949 & 22 &, 300 \\
& 2,00 &, 964 & 23 &, 543 \\
\hline
\end{tabular}

Fuente: elaboración propia

La prueba de Levene para la igualdad de varianzas (ver tabla 8) adquiere un valor de 0,103 por lo que se asumen la igualdad de varianza al ser superior a 0,05 . Esto hace pertinente la aplicación de la prueba t-student para determinar el grado de significatividad estadística de la varianza.

Una vez que los supuestos paramétricos han sido comprobados, los resultados de las pruebas aplicadas apuntan a diferencias estadísticamente significativas del grupo sometido al programa de intervención con respecto al grupo control en el post-test (tabla 8).

Tabla 8: Prueba de muestras independientes. Pre-test, test y post-test

\begin{tabular}{|c|c|c|c|c|c|c|c|c|c|c|}
\hline & & \multicolumn{2}{|c|}{$\begin{array}{c}\text { Prueba de Levene } \\
\text { de calidad de } \\
\text { varianzas }\end{array}$} & \multicolumn{7}{|c|}{ prueba t para la igualdad de medias } \\
\hline & & \multirow[b]{2}{*}{$\mathrm{F}$} & \multirow[b]{2}{*}{ Sig. } & \multirow[b]{2}{*}{$\mathrm{t}$} & \multirow[b]{2}{*}{$\mathrm{gl}$} & \multirow{2}{*}{$\begin{array}{c}\text { Sig. } \\
\text { (bilateral) }\end{array}$} & \multirow{2}{*}{$\begin{array}{c}\text { Diferencia de } \\
\text { medias }\end{array}$} & \multirow{2}{*}{$\begin{array}{c}\text { Diferencia } \\
\text { de error } \\
\text { estándar }\end{array}$} & \multicolumn{2}{|c|}{$\begin{array}{c}95 \% \text { de intervalo de } \\
\text { confianza de la } \\
\text { diferencia }\end{array}$} \\
\hline & & & & & & & & & Inferior & Superior \\
\hline $\begin{array}{l}\text { Pre } \\
\text { Test }\end{array}$ & $\begin{array}{l}\text { Se } \\
\text { asumen } \\
\text { varianzas } \\
\text { iguales }\end{array}$ & 3,182 & ,081 & $-2,307$ & 43 &, 026 & $-356,24802$ & 154,43502 & $-667,69591$ & $-44,80013$ \\
\hline & $\begin{array}{l}\text { No se } \\
\text { asumen } \\
\text { varianzas } \\
\text { iguales }\end{array}$ & & & $-2,324$ & 39,662 &, 025 & $-356,24802$ & 153,26961 & $-666,09965$ & $-46,39640$ \\
\hline Test & $\begin{array}{l}\text { Se } \\
\text { asumen } \\
\text { varianzas } \\
\text { iguales }\end{array}$ & 2,781 &, 103 & $-4,673$ & 43 &, 000 & $-850,68379$ & 182,05393 & $-1217,83053$ & $-483,53706$ \\
\hline & $\begin{array}{l}\text { No se } \\
\text { asumen } \\
\text { varianzas } \\
\text { iguales } \\
\end{array}$ & & & $-4,703$ & 40,576 &, 000 & $-850,68379$ & 180,86520 & $-1216,06451$ & $-485,30308$ \\
\hline $\begin{array}{l}\text { Post } \\
\text { Test }\end{array}$ & $\begin{array}{l}\text { Se } \\
\text { asumen } \\
\text { varianzas } \\
\text { iguales }\end{array}$ & 2,921 &, 095 & $-3,427$ & 43 & ,001 & $-539,35771$ & $\mid 157,38286$ & $-856,75049$ & $-221,96493$ \\
\hline & $\begin{array}{l}\text { No se } \\
\text { asumen } \\
\text { varianzas } \\
\text { iguales }\end{array}$ & & & $-3,451$ & 40,243 & ,001 & $-539,35771$ & 156,29451 & $-855,18129$ & $-223,53413$ \\
\hline
\end{tabular}

DISCUSIÓN

Fuente: elaboración propia

Una vez completado el análisis de los datos, iniciamos la discusión aportando al estado de la cuestión la certeza del aumento de la actividad física dentro de parámetros saludables, en la población de referencia. Considerando 
estudios anteriores (Tudor-Locke et al., 2011; Tudor-Locke et al., 2008), donde se establece el número de pasos necesarios (tabla 1) para que los sujetos del grupo de edad estudiado se consideren "activos", se ha detectado un aumento del volumen de actividad en los recreos de entre un $5 \%$ y un $6 \%$, durante las treinta sesiones que abarca el periodo de aplicación del programa.

Antes del programa, el recreo representaba entre el $12,85 \%$ y el $17,14 \%$ del total de actividad física considerado como saludable para la edad del alumnado (tabla 1). La aplicación del programa ha propiciado que los sujetos realicen en el recreo una actividad física equivalente a entre un $17,79 \%$ y un $23,72 \%$ de los pasos indispensables para que se produzca una mejora en la salud.

Tales resultados coinciden con los que reportan otros estudios (Ridgers et al., 2012; Sarkin et al., 1997) que han analizado la actividad física que realizan los estudiantes de Educación Primaria en los recreos escolares a partir de actividades propuestas por los investigadores. Sin embargo, cabe matizar que la mayoría de los estudios revisados se centran en las variables de género, curso, peso o niveles de intensidad de la práctica a través de acelerometría. Si bien, en sus recomendaciones plantean la conveniencia de organizar actividades para promover el aumento de la actividad física en periodos de recreo (Muñoz Caro, 2016).

Nuestra experiencia ha puesto de manifiesto que la asignatura de Educación Física puede ser el eje vertebrador de las alternativas de ocio saludables que aumenten la actividad física del alumnado en los recreos. Algo que ya apuntaba el trabajo de Faison-Hodge y Porretta (2004) en relación con el alumnado con necesidades educativas especiales.

Consideramos que el incremento de la actividad física en momentos de ocio tendrá más valor cuanto más se consolide en el tiempo la mejora. En este sentido, pasado un mes de la aplicación del programa hemos detectado que los sujetos han consolidado unos niveles de consolidación del hábito de actividad física muy aceptables, que se han concretado en un aumento medio de 300,44 pasos/día con respecto a los valores iniciales $(2.057,44$ pasos/día), siendo la actividad física media a los treinta días de 2.357,88 pasos. Esto equivale a un $38,07 \%$ de incremento mantenido de la actividad física en los recreos.

Al comparar estos resultados con los obtenidos por Guinhouya et al. (2006) y Ridgers et al. (2009) sobre hábitos saludables y niveles de actividad saludable en los recreos, encontramos que podríamos afirmar que el alumnado del programa ha desarrollado el hábito de realizar actividades físico motrices, en lugar de sedentarias, en los recreos.

\section{CONCLUSIONES}

Nuestra investigación permite afirmar que la aplicación del programa específico objeto de estudio tiene efectos en el nivel de actividad física observado durante los recreos, incidiendo positivamente sobre el volumen de 
actividad que realiza el alumnado en su tiempo libre. Hemos detectado un aumento significativo en la actividad física que el alumnado realiza durante los recreos escolares (en el contexto de referencia) después de haber experimentado el programa diseñado. También hemos constatado que, pasados treinta días de la aplicación del programa, el alumnado mantiene parte del incremento de actividad física que experimentó con la aplicación del programa, como evidencian los resultados de la comparación de la media de pasos entre grupo control y experimental (tabla 8).

La vinculación del programa a la asignatura de Educación Física ha sido determinante para la consecución de unos resultados tan favorables. Por ello, consideramos oportuno recomendar a la comunidad científica profundizar en esta línea de investigación. Especialmente cuando el estudio se oriente a la búsqueda de propuestas dirigidas a la promoción de la actividad física en momentos de ocio para personas en edad infantil y juvenil.

Cabe destacar que existe una escasa producción científica dirigida a estudiar la eficacia de estrategias que busquen aumentar la práctica física en el recreo escolar implementando un programa de intervención vinculado a la asignatura de Educación Física. Esta ausencia de estudios específicos está motivada, en gran medida, por la carencia de programas que relacionen al ocio en la edad infantil con los contenidos curriculares. Nuestra investigación también pretende contribuir al estado de la cuestión aportando un programa que ha demostrado ser efectivo para la población participante en el estudio (SánchezRivas y Vera-Estrada, 2013).

\section{REFERENCIA BIBLIOGRÁFICAS}

Aznar, S., Naylor, P. J., Silva, P., Pérez, M., Angulo, T., Laguna, M., ... LópezChicharro, J. (2011). Patterns of physical activity in Spanish children: a descriptive pilot study. Child: Care, Health and Development, 37(3), 322328. https://doi.org/10.1111/j.1365-2214.2010.01175.x

Beighle, A., Morgan, C. F., Le Masurier, G., \& Pangrazi, R. P. (2006). Children's physical activity during recess and outside of school. Journal of School Health, 76(10), 516-520. https://doi.org/10.1111/j.17461561.2006.00151.x

Escalante, Y., Backx, K., Saavedra, J. M., García-Hermoso, A., \& Domínguez, A. M. (2011). Relationship between daily physical activity, recess physical activity, age and sex in scholar of primary school, Spain. Revista española de salud pública, 85(5), 481-489. https://doi.org/10.1590/S113557272011000500007

Fairclough, S. J., Beighle, A., Erwin, H., \& Ridgers, N. D. (2012). School day segmented physical activity patterns of high and low active children. BMC Public Health, 12(1), 1-12. https://doi.org/10.1186/1471-2458-12-406

Faison-Hodge, J., \& Porretta, D. L. (2004). Physical activity levels of students with mental retardation and students without disabilities. Adapted Physical Activity Quarterly, 21(2), 139-152. https://doi.org/10.1123/apaq.21.2.139

Guinhouya, C. B., Hubert, H., Soubrier, S., Vilhelm, C., Lemdani, M., \& Durocher, A. (2006). Moderate-to-vigorous physical activity among children: 
discrepancies in accelerometry-based cut-off points. Obesity (Silver Spring, Md.), 14(5), 774-777. https://doi.org/10.1038/oby.2006.89

Haug, E., Torsheim, T., \& Samdal, O. (2010). Local school policies increase physical activity in Norwegian secondary schools. Health promotion international, 25(1), 63-72. https://doi.org/10.1093/heapro/dap040

Jennings-Aburto, N., Nava, F., Bonvecchio, A., Safdie, M., González-Casanova, I., Gust, T., \& Rivera, J. (2009). Physical activity during the school day in public primary schools in Mexico City. Salud pública de México, 51(2), 141147.

López Fernández, I., Pascual-Martos, C. A., \& Álvarez Carnero, E. (2013). Validation of a pedometer to Measure Physical Activity during school recess. Journal of sport and health research, 5(2), 167-178.

Martínez, J., Aznar, S., \& Contreras, O. (2015). El recreo escolar como oportunidad de espacio y tiempo saludable. Revista Internacional de Medicina y Ciencias de la Actividad Física y el Deporte, 15(59), 419-432. https://doi.org/10.15366/rimcafd2015.59.002

Martínez Martínez, J., Contreras Jordán, O. R., Lera Navarro, Á., \& Aznar Laín, S. (2012). Niveles de actividad física medido con acelerómetro en alumnos de $3^{\circ}$ ciclo de educación primaria: actividad física diaria y sesiones de educación física. Revista de Psicología del Deporte, 21(1), 117-123.

McKenzie, T. L., Crespo, N. C., Baquero, B., \& Elder, J. P. (2010). Leisure-time physical activity in elementary schools: Analysis of contextual conditions. Journal of School Health, 80(10), 470-477. https://doi.org/10.1111/j.17461561.2010.00530.x

Muñoz Caro, G. (2016). Promoción de actividad física durante los recreos escolares: trabajando con podómetros. Universidad Miguel Hernández, Elche. Recuperado a partir de http://dspace.umh.es//handle/11000/2786

OMS. (2017). Datos y cifras sobre obesidad infantil. Recuperado 3 de febrero de 2017, a partir de http://www.who.int/end-childhood-obesity/facts/es/

Pérez López, I. J., \& Delgado Fernández, M. (2013). Mejora de hábitos saludables en adolescentes desde la Educación Física escolar. Revista de educación, 360, 314-337. https://doi.org/10.4438/1988-592X-RE-2011360-113

Ridgers, N. D., Saint-Maurice, P. F., Welk, G. J., Siahpush, M., \& Huberty, J. (2011). Differences in physical activity during school recess. Journal of School Health, 81(9), 545-551. https://doi.org/10.1111/j.17461561.2011.00625.x

Ridgers, N. D., Salmon, J., Parrish, A.-M., Stanley, R. M., \& Okely, A. D. (2012). Physical activity during school recess: a systematic review. American journal of preventive medicine, 43(3), 320-328. https://doi.org/10.1016/j.amepre.2012.05.019

Ridgers, N. D., Stratton, G., \& Fairclough, S. J. (2005). Assessing physical activity during recess using accelerometry. Preventive medicine, 41(1), 102-107. https://doi.org/10.1016/j.ypmed.2004.10.023

Ridgers, N. D., Stratton, G., \& Fairclough, S. J. (2006). Physical activity levels of children during school playtime. Sports Medicine (Auckland, N.Z.), 36(4), 359-371. https://doi.org/10.2165/00007256-200636040-00005 
Ridgers, N. D., Stratton, G., \& McKenzie, T. L. (2010). Reliability and validity of the System for Observing Children's Activity and Relationships during Play (SOCARP). Journal of Physical Activity and Health, 7(1), 17-25. https://doi.org/10.1123/jpah.7.1.17

Ridgers, N. D., Tóth, M., \& Uvacsek, M. (2009). Physical activity levels of Hungarian children during school recess. Preventive Medicine, 49(5), 410412. https://doi.org/10.1016/j.ypmed.2009.08.008

Sánchez Rivas, E., \& Vera Estrada, F. (2013). El club de la W: ¡Hoy jugamos en clase!: Educación física, $6^{\circ}$ Educación Primaria. Sevilla: Wanceulen.

Sarkin, J. A., McKenzie, T. L., \& Sallis, J. F. (1997). Gender differences in physical activity during fifth-grade physical education and recess periods. Journal of Teaching in Physical Education, 17(1), 99-106. https://doi.org/10.1123/jtpe.17.1.99

Schneider, P. L., Crouter, S. E., \& Bassett, D. R. (2004). Pedometer measures of free-living physical activity: comparison of 13 models. Medicine and science in sports and exercise, 36(2), 331-335. https://doi.org/10.1249/01.MSS.0000113486.60548.E9

Stratton, G. (1999). Limits of agreement between 5 and 60 second heart rate recording intervals during school recess. Pediatric Exercise Science, 11, 253-254.

Stratton, Gareth, Ridgers, N. D., Fairclough, S. J., \& Richardson, D. J. (2007). Physical Activity Levels of Normal-weight and Overweight Girls and Boys During Primary School Recess. Obesity, 15(6), 1513-1519. https://doi.org/10.1038/oby.2007.179

Tudor-Locke, C., Craig, C. L., Beets, M. W., Belton, S., Cardon, G. M., Duncan, S., ... Blair, S. N. (2011). How many steps/day are enough? for children and adolescents. The International Journal of Behavioral Nutrition and Physical Activity, 8, 78. https://doi.org/10.1186/1479-5868-8-78

Tudor-Locke, C., Hatano, Y., Pangrazi, R. P., \& Kang, M. (2008). Revisiting “how many steps are enough?". Medicine and science in sports and exercise, 40(7S), S537-S543. https://doi.org/10.1249/MSS.0b013e31817c7133

Tudor-Locke, C., Johnson, W. D., \& Katzmarzyk, P. T. (2010). AccelerometerDetermined Steps per Day in US Children and Youth. Medicine \& Science in Sports \& Exercise: Official Journal of the American College of Sports Medicine, $42(12)$, 2244-2250. https://doi.org/10.1249/MSS.0b013e3181e32d7f

Tudor-Locke, C., McClain, J. J., Hart, T. L., Sisson, S. B., \& Washington, T. L. (2009). Pedometry methods for assessing free-living youth. Research quarterly for exercise and sport, 80(2), 175-184. https://doi.org/10.1080/02701367.2009.10599551

World Health Organization. (2010). Global recommendations on physical activity for health. Recuperado a partir de http://www.ncbi.nlm.nih.gov/books/NBK305057/

Referencias totales / Total references: 31 (100\%) Referencias propias de la revista / Journal's own references: 1 (3,2\%)

Rev.int.med.cienc.act.fís.deporte - vol. 18 - número 72 - ISSN: 1577-0354 